\title{
A continuum damage mechanics model for 2-D woven oxide/oxide ceramic matrix composites under cyclic thermal shocks
}

\author{
Zhengmao Yang ${ }^{\mathrm{a}, *}$, Hui Lui ${ }^{\mathrm{b}}$ \\ ${ }^{a}$ Institute of Mechanics, Chinese Academy of Sciences, Beijing, China \\ ${ }^{\mathrm{b}}$ School of Aerospace Engineering, Tsinghua University, Beijing, China
}

\section{A R T I C L E I N F O}

\section{Keywords:}

Ceramic matrix composites (CMCs)

Thermal shock

Mechanical properties

Thermomechanical damage evolution

Matrix cracks

\begin{abstract}
A B S T R A C T
Oxide/oxide-ceramic matrix composites (ox/ox-CMCs) are excellent candidates for material applications in hightemperature and oxidizing environments, which exhibit complex couplings between the thermal and mechanical fields. Uniaxial tensile tests are conducted for $0^{\circ} / 90^{\circ}$ and $\pm 45^{\circ}$ fiber orientation of the thermal shocked ox/oxCMCs. The study reveals that the anisotropy properties of the thermal shocked ox/ox-CMCs result in different degrees of thermomechanical damage, which are reflected in the degeneration of the modulus. Based on the detailed experiments and analysis, a cyclic thermal shock-induced thermomechanical damage model of the 2-D woven ox/ox-CMCs is proposed, incorporating two tensile scalar damage parameters, and one shear scalar damage parameter. The model will contribute to predicting the thermomechanical damage evolution throughout the composite structure under thermal transient conditions. In addition, the model was validated with numerical simulation to demonstrate the damage model performance, which is relatively crucial in damage tolerance analysis in the aeronautical industry.
\end{abstract}

\section{Introduction}

Oxide/oxide-ceramic matrix composites (ox/ox-CMCs) exhibit high strength, low density, high-temperature stability, and excellent corrosion resistance, which can meet the thermomechanical property requirements of various high-temperature applications [1-3]. Therefore, CMCs are good candidates for aeronautical industry applications, such as thermal combustors, nozzle guide vanes, and flame stabilizers in gas turbines. The mechanical responses of CMC structures are always related to the factors present in the combustion environment, such as cyclic thermal shocks, thermal aging, water vapor, and corrosion [4]. High-temperature gradients under harsh environments will result in excessive thermal stresses and accompanying microstructural changes, which accelerate premature damage and failure of the composite structures. Consequently, it is of considerable significance to investigate the residual mechanical behavior and thermomechanical damage mechanism of ox/ox-CMCs under thermal transient loading and to quantitatively describe the thermomechanical damage evolution of materials.

Several studies have been performed on understanding and describing the mechanical behavior of these composites at high temperatures [5,6], notably considering the effect of matrix cracks and interfaces on the mechanical properties of composites. Singh and Wang
$[7,8]$ discovered that the main thermal shock damage mechanisms are matrix cracking and delamination for three types of continuous fiberreinforced CMCs after thermal shocks when observing the mechanical behavior. Kagawa [9] investigated the development of thermomechanical damage in the SiC-fiber-reinforced SiC-matrix composites fabricated by the chemical vapor infiltration (CVI) process under thermal shocks, and the experimental results demonstrated that the crack initiation is related to the critical temperature. Kastritseas et al. $[10,11]$ observed multiple matrix cracking in cross-ply Nicalon/calcium aluminosilicate (CAS) CMCs after thermal shocks, and proposed a semiempirical formula to predict the critical thermal shock temperature for crack initiation. Salekeen et al. [12] studied the degradation of mechanical properties of the SiC/SiNC CMCs manufactured by the polymer impregnation and pyrolysis (PIP) process owing to thermal shocks, the results show that both the elastic modulus and strength decrease by about $20 \%$ after five thermal shock cycles. Mei and Cheng [13] introduced the time dependence and stress dependence of cyclic fatigue (both thermal and mechanical) damage of $\mathrm{C} / \mathrm{SiC}$ composites and experimentally discussed the effects of the fatigue cycles and the fatigue stress levels on the tensile behavior of composites. Leanos and Prabhakar [14] investigated the compressive properties of the original and thermal shocked 2-D C/C composites, which shows that the compressive strength and stiffness decrease as the thermal shock temperature

\footnotetext{
* Corresponding author.

E-mail address: zmyang@imech.ac.cn (Z. Yang).
} 
increases. Udayakumar et al. [15] quantified the interfacial bond strength in $\mathrm{SiC}_{\mathrm{f}} / \mathrm{SiC}$ composites and reported the materials mechanical properties before and after thermal shocks. Zhang et al. [16] studied the tensile properties of 2.5-D C/SiC composites reinforced by woven T-300 carbon fiber in the warp and weft direction influenced by the thermal cycles. They found that the modulus decreases with increasing thermal cycles, and the degradation of modulus in the warp direction is $37 \%$ for the as-received material, whereas that of the weft modulus is only $18 \%$. Triantou et al. [17] evaluated the performance of carbon-bonded fiber composites and CMCs on thermal shock tests through scanning electron microscopy (SEM) and energy dispersive spectroscopy (EDS). Unfortunately, little attention has been paid to characterizing and quantifying the thermal shock-induced thermomechanical damage of ox/oxCMCs, and there is no reliable model to investigate and predict the thermomechanical damage evolution for ox/ox-CMCs under cyclic thermal shocks.

The damage mechanic approaches to formulating the constitutive laws for the damaged materials can be classified into two categories: the micromechanical approaches and the macroscopic approaches based on continuum damage mechanics (CDM) [18-20]. Micromechanical approaches have been developed over the past few decades, which provide essential insights for analyzing the mechanism of microstructural damage (i.e., fiber pullout, matrix cracking, debonding). However, they are difficult to extend to the materials with laminates and to the macro-scale in a generic manner [21,22]. The CDM approaches are generally attractive when modeling damage initiation and development for composites, as the damage is the primary energy dissipation source in these materials $[23,24]$. The damage evolution laws can be governed by thermodynamic forces and calibrated using extensive experimental data. Recently, Yang and Yuan [25,26] investigated cyclic thermal shock-induced thermomechanical damage in CMCs and found that the micro-cracks in the matrix induced by thermal stresses mainly result in macroscopic damage during thermal transient conditions. The material degradation is characterized by a reduction in elastic modulus and driven by the elastic strain energy. A thermomechanical damage model was developed based on experiments within the framework of CDM. However, the model is based on the isotropic assumption and neglects the effect of anisotropy on the damage evolution for the composites. As the damage in ox/ox-CMCs involves several mechanisms that are highly anisotropic, it is of importance to take into account the effects of anisotropy on the mechanical properties when describing the damage in the thermal shocked CMCs.

In the present work, the mechanical behaviors of $0^{\circ} / 90^{\circ}$ and $\pm 45^{\circ}$ fiber orientated 2-D woven oxide/oxide CMCs related to the thermal shock temperature and cumulative number of thermal shock cycles are investigated. Meanwhile, the evolution of microscopic damage mechanisms is also experimentally analyzed. A thermomechanical damage evolution model for ox/ox-CMCs under cyclic thermal shocks is proposed using the framework of CDM and thermodynamics, in addition, the verification and validation of this model are realized through numerical simulations in the ABAQUS/Standard integrated environment.

\section{Materials and experiments}

\subsection{Materials}

The 2-D woven ox/ox-CMCs were manufactured using uncoated Nextel ${ }^{\mathrm{TM}} 610\left(99 \% \alpha-\mathrm{Al}_{2} \mathrm{O}_{3}\right)$ fibers and $\mathrm{Al}_{2} \mathrm{O}_{3}-\mathrm{SiO}_{2}-\mathrm{ZrO}_{2}$ matrix. The Nextel $^{\mathrm{TM}} 610$ fibers with a diameter of $10 \mu \mathrm{m}$ account for approximately $44 \%$ of the total material volume, and the density of fibers is $4.0 \mathrm{~g} / \mathrm{cm}^{3}$. The matrix has a chemical composition of $85 \mathrm{wt} \% \mathrm{Al}_{2} \mathrm{O}_{3}$ and $15 \mathrm{wt} \%$ 3YSZ ( $3 \mathrm{~mol} \%$ Yttria-stabilized monoclinic and tetragonal $\mathrm{ZrO}_{2}$ ), the density of which is $4.2 \mathrm{~g} / \mathrm{cm}^{3}$. First, the fibers woven in 8-harness satin (8HS) were impregnated with the paste-like $\mathrm{Al}_{2} \mathrm{O}_{3}$ slurry by blade coating. Then the 8 plies of fiber layers were infiltrated with 3YSZ-sol, stacked in $0^{\circ} / 90^{\circ}$ order. Finally, the sintering process was performed at

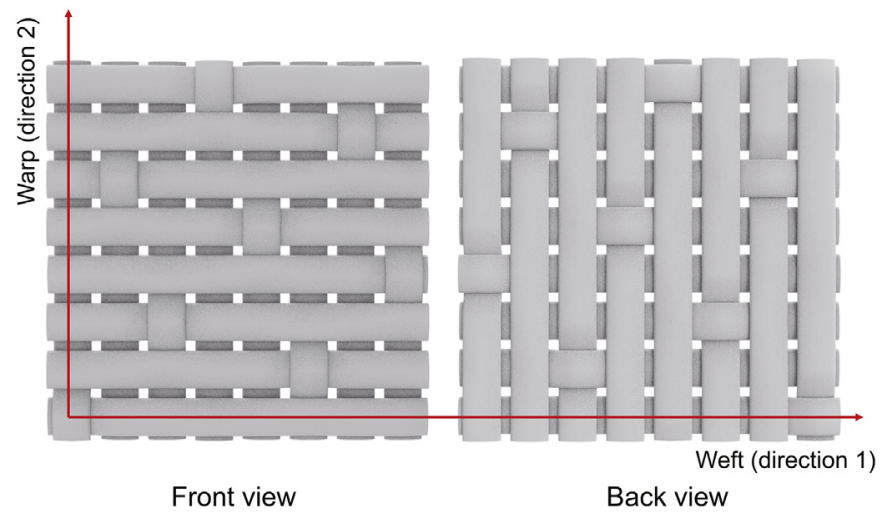

Fig. 1. Schematic of 8-harness satin weave in ox/ox-CMCs.

a temperature of $1000-1300{ }^{\circ} \mathrm{C}$ for $5 \mathrm{~h}$. The finished composite panel was $2.8 \mathrm{~mm}$ in thickness, and the total open porosity, determined by the mercury intrusion method, is $27.6 \%$. A schematic representation of $8 \mathrm{HS}$ weave ply is shown in Fig. 1.

The overall microstructure of the original ox/ox-CMCs is presented in Fig. 2. It illustrates that some residual pores and extensive microcracking appear in the matrix. The pores arise during the material processing, which have been created by gas trapped with the matrix infiltrated fiber preform. Because of the stress concentration occurring at the corner of the pores, the micro-cracks will initiate from the corners of the pores when suffering the cyclic thermal shocks. The excessive shrinkage of materials is caused by the high-temperature gradients during material fabrication, and the degree of shrinkage between fiber and matrix is mismatched owing to the different coefficients of thermal expansion (CTE) in fiber and matrix, which leads to the existence of micro-cracking. These inherent pores and micro-cracking have a significant effect on the mechanical properties, as discussed in Refs. $[26,27]$.

\subsection{Cyclic thermal shock tests}

The cyclic thermal shock tests were performed at the maximum thermal shock temperature of $1100^{\circ} \mathrm{C}$, which is slightly below the maximum potential operating temperature of the ox/ox-CMCs. During each thermal shock cycle, specimens were elevated in the center of the muffle furnace for about $10 \mathrm{~min}$ at $1100^{\circ} \mathrm{C}$. Once the sample and the environment in the furnace reached a sufficient thermal balance, the sample was immediately taken out and placed in a distilled water bath at a temperature of $20^{\circ} \mathrm{C}$. After the water quenching, the specimens were dried in air at room temperature before putting back into the furnace for another thermal shock cycle. Further details for the cyclic thermal shock tests can be found in Refs. [25,27]. After finished the preselected thermal shock number, SEM was used to examine the microstructural evolution of the materials.

\subsection{Mechanical test}

To represent the in-plane mechanical properties of the ox/ox-CMCs under cyclic thermal shocks, the residual modulus in $0^{\circ} / 90^{\circ}$ and $\pm 45^{\circ}$ fiber orientated composites were measured using uniaxial tensile tests at room temperature. The specimens from the same batch were cut into the standard shape of $160 \mathrm{~mm} \times 20 \mathrm{~mm}$ with a gage length of $15 \mathrm{~mm}$ by a water jet, as shown in Fig. 3. For each specimen, a strain gage rosette was affixed to the gage section of the specimen to measure the tensile strains and its corresponding transversal strains. All the tensile tests were performed by strain control in a servo-controlled MTS mechanical testing machine [25], with constant strain rate $10^{-4} / \mathrm{s}$ ensuring quasistatic loading conditions.

Owing to the limitations of the test conditions, the in-plane shear 

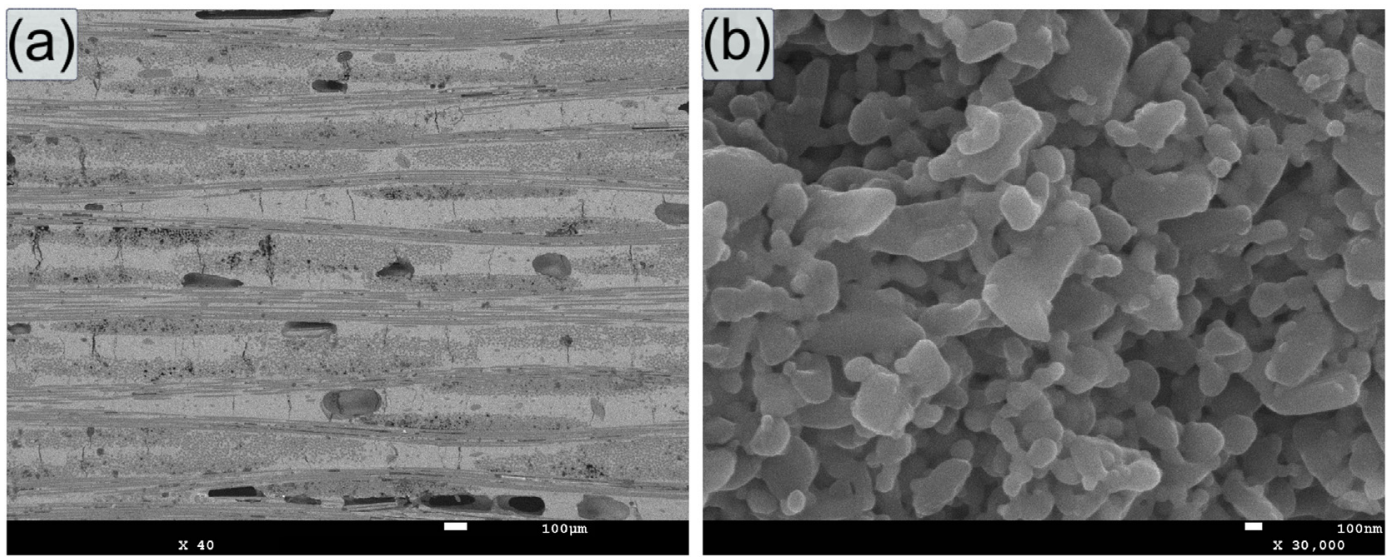

Fig. 2. SEM observations of a cross-section through the as-received ox/ox-CMCs. (a) Micro-pores and sintering shrinkage cracks in the matrix (perpendicular to the plies of the matrix). (b) Nano-pores of the matrix, enabling crack initiation and deflection at the fiber/matrix interface.

test was not performed to evaluate the pure shear mechanical properties after cyclic thermal shocks. Therefore, the shear properties were determined by tensile test data of $\pm 45^{\circ}$ off-axis specimens, in accordance with the ASTM standard D3518 [28]. The in-plane shear modulus can be determined based on the following relationship [27,29]:

$G_{12}=\frac{\sigma_{x}}{2\left(\varepsilon_{x}-\varepsilon_{y}\right)}$,

where $\sigma_{x}$ is tensile stress of the $\pm 45^{\circ}$ off-axis specimens, $\sigma_{x}=F / A$, and $A$ is the cross-sectional area for the specimen in the tensile direction. The axial strain $\varepsilon_{x}$ and the transverse strain $\varepsilon_{y}$ are measured using the strain gage rosette.

\section{Characterization of residual mechanical properties}

\subsection{Microstructural evolution}

To study the damage mechanism of the ox/ox-CMCs under cyclic thermal shocks, a large number of detailed SEM observations were performed, and two kinds of damage mechanisms can be observed.

(i) Delamination in the interface of fiber and matrix, which is caused by high-temperature gradients and different CTEs for the fiber and matrix.

(ii) Micro-cracks, along with the thickness of the matrix, caused by the tensile stress. As the matrix between the fiber bundle is brittle and stiffer, and the matrix has larger built-in pores than the fiber bundle, thus the matrix begins to crack first. The corresponding crack grows between and inside the fiber bundles at the transverse plane under the thermomechanical loading, which is oriented by the fibers, as shown in Fig. 4.
It seems that the delamination is independent of cyclic thermal shock numbers, but the matrix crack density increases with the thermal shock cycle number, which leads to the continuous degeneration of the material property with cyclic thermal shocks. More specifically, when suffering only a limited number of thermal shock cycles (the shock cycle number is about 8 for the present materials and the thermal shock temperature $T_{\text {shock }}=1100^{\circ} \mathrm{C}[25]$ ), the rate of increase of the microcrack density slows down, as the fiber reinforcements change the crack initiation and crack propagation behavior. Ultimately, the thermomechanical damage of the ox/ox-CMCs reaches a saturation level (characteristic damage state (CDS)).

\subsection{Mechanical property characterization}

As analyzed previously, the increase of micro-crack density results in the degeneration of mechanical properties, thus it is essential to investigate the residual mechanical property of the ox/ox-CMCs under cyclic thermal shocks materials. The $0^{\circ} / 90^{\circ}$ and $\pm 45^{\circ}$ fiber orientations of the composites are chosen to conduct tensile tests as the mechanical response of the ox/ox-CMCs depends on the applied load direction. The mechanical behavior in the $0^{\circ} / 90^{\circ}$ orientation is dominated by fibers in the in-plane loading direction. The load in the $\pm 45^{\circ}$ off-axis direction is transferred to the fiber by the matrix, and the matrix dominates the stiffness and strength behavior in the $\pm 45^{\circ}$ orientation.

For a single thermal shock with different thermal shock temperatures, the elastic modulus $E_{1}, E_{2}$ and in-plane shear modulus $G_{12}$ of the ox/ox-CMCs decrease with thermal shock temperature $T_{\text {shock }}$ increases, the drop in $G_{12}$ reaches $18.5 \%$, whereas $E_{1}$ and $E_{2}$. are reduced by $14 \%$ and $8 \%$, respectively, as indicated in Fig. 5(a). The experimental results show that the elastic modulus $E_{1}$ and $E_{2}$ differ by as much as $22 \%$, although the material is a $0^{\circ} / 90^{\circ}$ symmetrical laminate. There are two possible explanations for the difference: (i) The edge effects, because

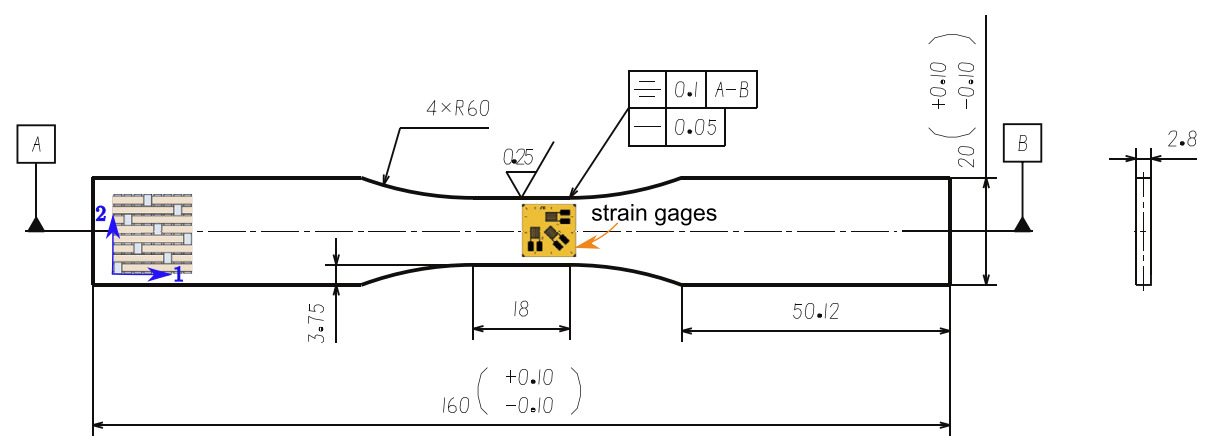

Fig. 3. Schematic illustration of the specimen geometry, a strain gage rosette is used for room-temperature mechanical property measurement. 

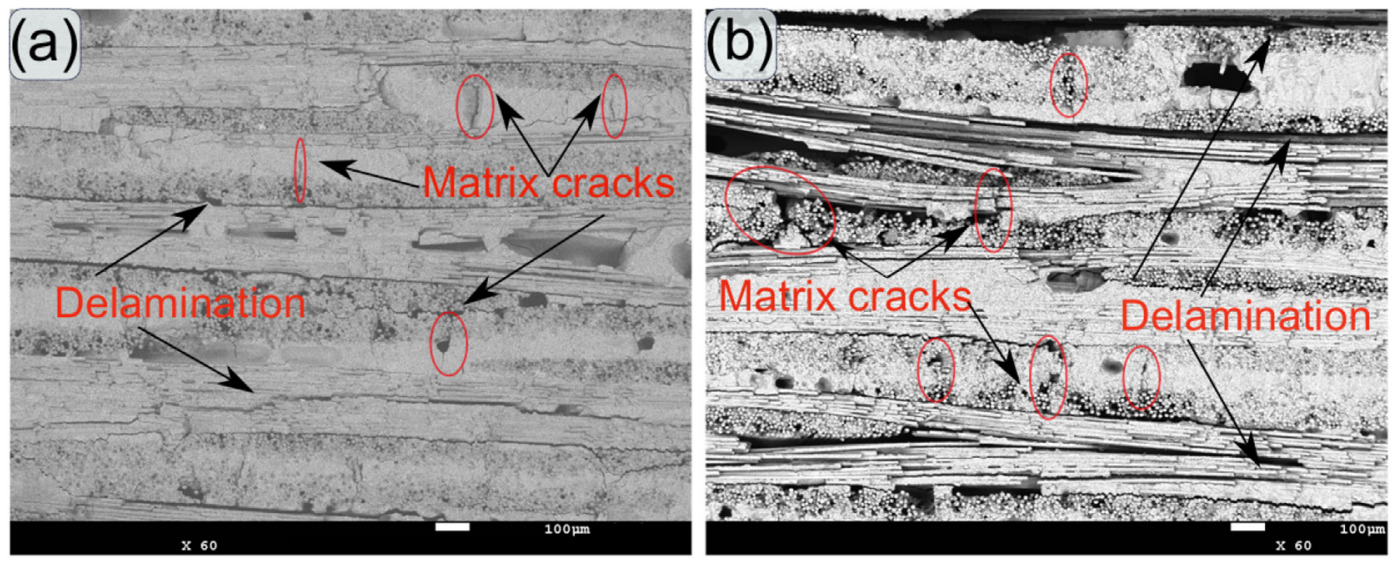

Fig. 4. SEM micrographs of a cross-section through the ox/ox-CMCs after thermal shocks. (a) After one thermal shock, micro-cracks initiated from the corners of nano-pores of the matrix. (b) After eight thermal shocks, transverse crack density continues up to a saturation point and generates long cracks.

the width of the gage section is lower than the length [30], and the fiber braiding mode leads to inconsistencies in the fiber distribution in length and width; (ii) The coefficient of heat conduction in direction 1 is larger than that in direction 2, causing higher thermal stress when suffering thermal shocks, and there are more thermal shock-induced microcracks in the 1 direction, which finally result in more decrease of $E_{1}$. The difference between the properties of warp and weft directions is the case in most of the composites with woven fibers [31].

The difference in $E_{1}, E_{2}$, and $G_{12}$ experimentally verifies that the orthogonal material property of the ox/ox-CMCs affects the material degeneration. Moreover, the stiffness reduction of the three principal directions occurs when the shock temperature reaches $400{ }^{\circ} \mathrm{C}$ as this is the thermal shock temperature threshold. Furthermore, the elastic modulus reaches a saturation level, as $T_{\text {shock }}$ exceeds $1000{ }^{\circ} \mathrm{C}$ and even higher temperatures.

Fig. 5(b) shows the residual modulus of the ox/ox-CMCs under cyclic thermal shocks, with the thermal shock temperature of $1100^{\circ} \mathrm{C}$. The modulus $E_{1}, E_{2}$, and $G_{12}$ decrease with cyclic thermal shock number increases, and the overall reduction of $E_{1}$ is higher than $E_{2}$, as the change rate of $E_{2}$ is lower compared with $E_{1}$, which displays the same phenomenon as the materials under single thermal shock. Here $E_{1}$ and $E_{2}$ reach saturation after eight thermal shocks, whereas $G_{12}$ approaches the final state after four thermal shocks. As the crack grows with the number of cyclic thermal shocks, the release of strain energy will reduce the stress concentration occurring at the corner of pores, and when the crack tip reached another pore between fiber bundles, cracks will be stopped, thus it seems natural for the modulus to eventually become

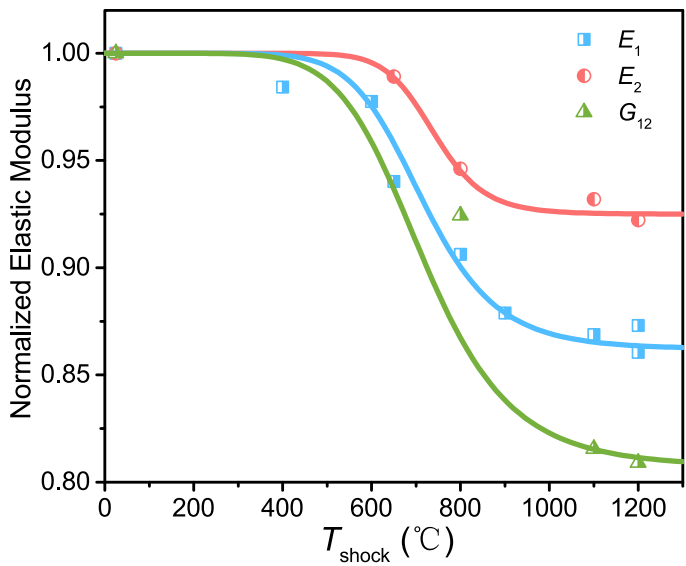

(a) Residual modulus as a function of an increasing thermal shock temperature $T_{\text {shock }}$. saturated.

\subsection{The modeling of thermomechanical damage}

The thermomechanical damage affects the mechanical behavior of CMCs considerably, which causes degradation in the elastic modulus as the damage accumulates and grows [32,33]. The plasticity is neglected when modeling the mechanical behavior of the ox/ox-CMCs, as the materials exhibit elastic-brittle behavior. More essentially, the damage can be related to elastic strain energy under thermomechanical conditions. Consequently, to predict the thermomechanical damage initiation and evolution of the cyclic thermal shocked ox/ox-CMCs, the elastic thermal-damage law was introduced into the orthotropic constitutive model to describe the material response behavior of the ox/ox-CMCs under cyclic thermal shocks.

\subsection{Constitutive model}

A constitutive description of damage is important in quantifying the development of thermomechanical damage for the cyclic thermal shocked ox/ox-CMCs. In the present work, the orthotropic damage is considered for simplicity according to the orthotropic symmetry of the materials, and it is assumed that the orthotropy of the materials remains unaltered during the cyclic thermal shocks, which can be proved by experiments [25].

Using the stiffness tensor of the damaged material $\tilde{\mathscr{E}}$, the representation of the actual stress state $\sigma$ experienced by the damaged

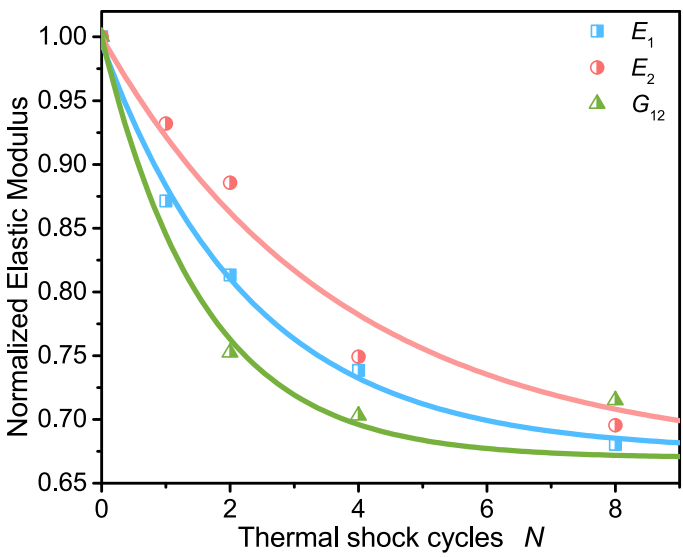

(b) Residual modulus as a function of an increasing cumulative number of thermal shock cycles, $N$.

Fig. 5. Residual modulus evolution of the thermal shocked ox/ox-CMCs. 
material and the von Mises effective stress $\tilde{\sigma}$ are defined as

$\sigma=\tilde{\mathscr{E}}: \varepsilon, \quad \tilde{\sigma}=\mathscr{E}: \varepsilon$

where $\sigma=\left[\sigma_{1} \sigma_{2} \sigma_{6}\right]$ and $\varepsilon=\left[\varepsilon_{1} \varepsilon_{2} \varepsilon_{6}\right]$ considering the orthotropy of the materials.

Combining Eq. (2), the relationship between the effective stress $\tilde{\sigma}$ and the actual stress $\sigma$ can be obtained:

$\tilde{\sigma}=\mathscr{E}^{-1}: \sigma$,

where the damaged stiffness matrix $\tilde{\mathscr{E}}$ can be expressed by the internal state variables $\boldsymbol{D}$

$\tilde{\mathscr{E}}=\tilde{\mathscr{E}}(\boldsymbol{D}, \mathscr{E})$.

On account of the hypotheses and CDM, the scalar damage variables $D_{1}, D_{2}, D_{6}$ constitute the internal state variables, as $\boldsymbol{D}=\left[D_{1} D_{2} D_{6}\right]^{\top}$, which characterize the damage in the different principal directions [34,35],

$D_{1}=1-\frac{E_{1}}{E_{1}^{0}}, \quad D_{2}=1-\frac{E_{2}}{E_{2}^{0}}, \quad D_{6}=1-\frac{G_{12}}{G_{12}^{0}}$,

where $E_{1}, E_{2}$, and $G_{12}$ are the modulus of the damaged materials and the superscript 0 means the original state of the ox/ox-CMCs. As for the internal variables, $D_{1}$ quantifies $0^{\circ}$ fiber-direction stiffness degradation damage, $D_{2}$ represents $90^{\circ}$ fiber-direction stiffness degradation damage, and $D_{6}$ represents in-plane shear damage. The experimental observations generate the database in subsection 3.2 for identifying the damage values.

Then the $\mathscr{E}^{-1}$ in Eq. (3) can be expressed by the damage $\boldsymbol{D}$, as

$\tilde{\mathscr{E} \mathscr{E}} \tilde{-1}^{-1}=\left[\begin{array}{ccc}\frac{1}{1-D_{1}} & 0 & 0 \\ 0 & \frac{1}{1-D_{2}} & 0 \\ 0 & 0 & \frac{1}{1-D_{6}}\end{array}\right]$.

Assuming the isothermal deformation process in orthotropic materials, the thermodynamic potential function can be reduced to the elastic strain energy expressed in terms of the material properties and damage variables,

$$
\begin{aligned}
\rho \Phi= & \sigma: \tilde{E}^{-1}: \sigma=\frac{1}{2} \frac{\sigma_{1}^{2}}{E_{1}^{0}\left(1-D_{1}\right)}-\frac{\nu_{12}^{0} \sigma_{1} \sigma_{2}}{E_{1}^{0}}+\frac{1}{2} \frac{\sigma_{2}^{2}}{E_{2}^{0}\left(1-D_{2}\right)} \\
& +\frac{1}{2} \frac{\sigma_{6}^{2}}{G_{12}^{0}\left(1-D_{6}\right)}
\end{aligned}
$$

where $\nu_{12}^{0}$ is the primary in-plane Poisson ratio and the compliance coefficient $-\nu_{12} / E_{1}$ is assumed to be constant. Then, the state law can be written as the derivative of $\Phi$ versus the stress tensor $\sigma$ :

$\varepsilon=\rho \frac{\partial \Phi}{\partial \sigma}$

where $\varepsilon$ denotes the elastic strain tensor.

\subsection{Damage evolution for a single thermal shock}

For a single thermal shock with different thermal shock temperatures $T_{\text {shock}}$, the strain energy density release rate, $Y_{i}$, as the thermodynamic force is used to govern the thermal shock damage evolution, as described in the previous study [25]. According to the thermodynamics of CDM and definition of the dissipation potential, the thermodynamic forces associated with the dissipative mechanisms can be derived from the energy release rates [36]:
$Y_{1}=\rho \frac{\partial \Phi}{\partial D_{1}}=\frac{1}{2 E_{1}^{0}} \frac{\sigma_{1}^{2}}{\left(1-D_{1}\right)^{2}}=\frac{\tilde{\sigma}_{1}^{2}}{2 E_{1}^{0}}$

$Y_{2}=\rho \frac{\partial \Phi}{\partial D_{2}}=\frac{1}{2 E_{2}^{0}} \frac{\sigma_{2}^{2}}{\left(1-D_{2}\right)^{2}}=\frac{\tilde{\sigma}_{2}^{2}}{2 E_{2}^{0}}$

$Y_{6}=\rho \frac{\partial \Phi}{\partial D_{6}}=\frac{1}{2 G_{12}^{0}} \frac{\sigma_{6}^{2}}{\left(1-D_{6}\right)^{2}}=\frac{\tilde{\sigma}_{6}^{2}}{2 G_{12}^{0}}$.

Assuming that the thermal stress $\sigma_{\text {th }}$ that causes damage to the material is equivalent to the effective stress, $\sigma_{\text {th }}=\tilde{\sigma}$, the strain energy density release rate can be obtained:

$Y_{i}=\frac{\left(\sigma_{\mathrm{th}, i}\right)^{2}}{2 E_{i}^{0}}, \quad i=1,2,6$.

The second principle of thermodynamics is satisfied, which is written as the Clausius-Duhem inequality:

$\sigma_{i j} \dot{\varepsilon}_{i j}^{\mathrm{p}}-\dot{w}_{s}+Y_{i j} \dot{D}_{i j}-\frac{q_{i} T_{, i}}{T} \geq 0$.

As the dissipation is positive, and the thermomechanical damage is considered to be the only dissipative phenomenon in the materials, the damage evolution laws should obey the following inequality: $\dot{D}_{i j} \geq 0$.

It is assumed that the damage is uniformly distributed through the thickness of the composite, thus the definition of the potential function for the thermal shock damage is [36],

$\mathscr{\mathscr { F }}_{i}^{D}=Y_{i}-\mathscr{Z}_{i}(D)$,

where $\mathscr{Z}_{i}$ is defined as the material resistance against damage which has the form:

$\mathscr{Z}_{i}=Y_{\mathrm{th}, i}+\frac{1}{b_{i}} \ln \left(\frac{D_{i}^{\mathrm{sat}}}{D_{i}^{\mathrm{sat}}-D_{i}}\right)$,

where $Y_{\mathrm{th}, i}$ is the threshold of the energy density release rate, $b_{i}$ is the model parameter, and $D_{i}^{\text {sat }}$ represents the saturation of the thermal shock damage. The damage evolution law is [36],

$\dot{D}_{i}=\dot{\lambda} \frac{\partial \mathscr{F}_{i}^{D}}{\partial Y_{i}}=\dot{\lambda}$,

where $\dot{\lambda}$ is the damage multiplier: according to the Kuhn-Tucker condition, it is non-negative for thermal shock conditions. The evolution of the thermal shock damage becomes,

$\dot{D}_{i}=D_{i}^{\mathrm{sat}} b_{i} \exp \left[-b_{i}\left\langle Y_{i}-Y_{\mathrm{th}, i}\right\rangle\right] \dot{Y}_{i}$,

where $\langle\cdot\rangle$ are the Macauley brackets. Integrating Eq. (15) over $\left[Y_{\mathrm{th}, i}, Y_{i}\right]$, the explicit thermal shock-induced thermomechanical damage expression is obtained:

$D_{i}=D_{i}^{\mathrm{sat}}\left\{1-\exp \left[-b_{i}\left\langle Y_{i}-Y_{\mathrm{th}, i}\right\rangle\right]\right\}$.

The initial damage resistance $Y_{\mathrm{th}, i}$, the parameter $b_{i}$, and the saturation damage $D_{i}^{\text {sat }}$ determine the thermomechanical damage caused by a single thermal shock with different thermal shock temperatures. The model parameters obtained from the experiments are listed in Table 1.

Fig. 6 illuminates the damage evolution of the ox/ox-CMCs subjected to a single thermal shock with varying thermal shock temperature $T_{\text {shock}}$. When the strain energy density release rate $Y_{i}$ reaches the threshold value $Y_{\mathrm{th}, i}$, the thermal shock-induced thermomechanical damage increases rapidly, then reaches the saturation stage, owing to the decrease of the strain energy damage rate in the maximum principal

Table 1

Thermomechanical damage model parameters of ox/ox-CMCs.

\begin{tabular}{llll}
\hline Model parameters & $D_{1}$ & $D_{2}$ & $D_{6}$ \\
\hline$D_{i}^{\text {sat }}$ & 0.139 & 0.073 & 0.192 \\
$Y_{\text {th }, i}(\mathrm{MPa})$ & 0.150 & 0.150 & 0.090 \\
$b_{i}$ & 6.97 & 18.92 & 41.26 \\
\hline
\end{tabular}




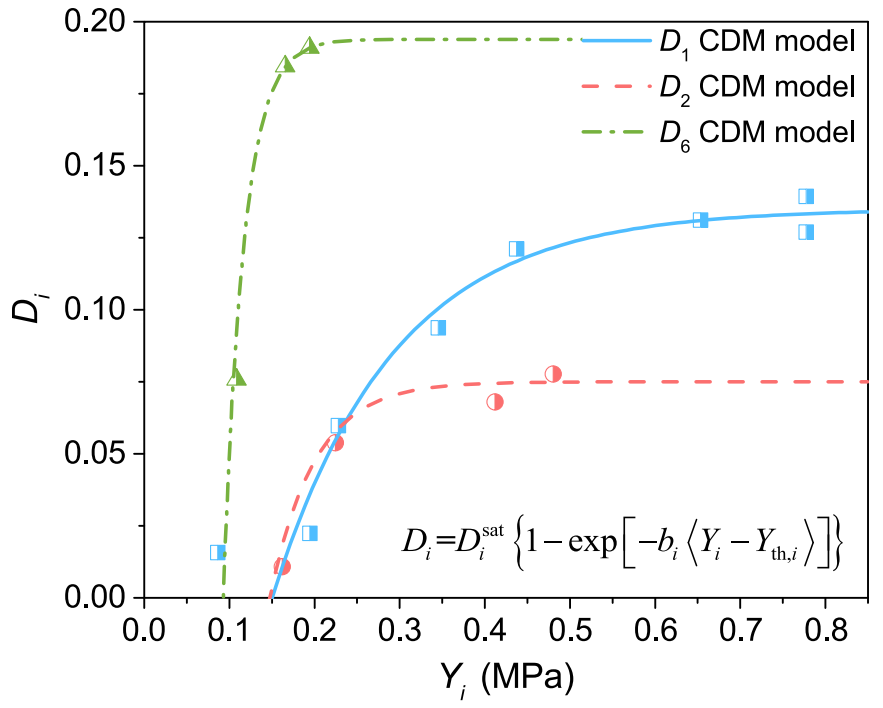

Fig. 6. Damage evolution with the strain energy density release rate for the ox/ ox-CMCs under a single thermal shock with different shock temperatures $T_{\text {shock }}$. Symbols denote experimental results, whereas the curves are predictions from the CDM model.

stress plane of the materials. Compared with the growth rate of damage in plane direction 1 , the growth rate of in-plane shear damage $D_{6}$ is much higher, which demonstrates that the thermomechanical damage in the matrix is serious, as the shear property of lamina is affected mainly by matrix. This damage evolution is physically reasonable. On the other hand, $D_{1}$ and $D_{2}$ show different saturation values, even though the change rates of $D_{1}$ and $D_{2}$ are almost the same. It may be caused by the influence of the specimen size and edge effect, as well as that the thermal stress gradients in two directions are different due to the different thermal conductivities in the process of thermal shocks.

\subsection{Damage evolution for cyclic thermal shocks}

The cyclic thermal shock process is thermal mechanical fatigue in nature, which may be regarded as energy-dissipative processes in CMCs. Considering that the damage is cumulative with the dissipation of energy, the energy methods become imperative for characterizing damage evolution during cyclic thermal shocks [25]. Then the strain energy in the damaged materials $\mathscr{U}$ can be rewritten as

$\mathscr{U}_{i}=\mathscr{U}_{0}^{i}\left(1-D_{i}\right)^{2}$

with

$\mathscr{U}_{0}=\frac{1}{2}\left[\frac{\alpha T_{\text {shock }}}{(1-\nu)} \psi\left(B_{\mathrm{i}}\right)\right]^{2} E_{0} f(\eta)$,

where $\alpha$ denotes the CTE of composites, $\psi\left(B_{\mathrm{i}}\right)$ is a function of the Biot modulus related to the maximum stress and rate of heat transfer, and $\mathscr{U}_{0}$ is the strain energy per unit volume for the original ox/ox-CMCs.

The evolution of the strain energy in the materials under cyclic thermal shocks is shown in Fig. 7. Obviously, with the increase in the number of shock cycles $N$, the strain energy of the materials will decrease as the cyclic thermal shocks cause the damage $D$ in the materials. In other words, the damage increment $\Delta D$ from cycle to cycle represents the strain energy dissipation, as the damage is the only energy consumer. To illustrate the relationship between the damage and the energy dissipation, the energy dissipation can be defined as

$\Pi_{N}^{i}=\sum_{k=1}^{N}\left(\mathscr{U}_{k-1}^{i}-\mathscr{U}_{k}^{i}\right)$

where $\mathscr{U}_{k}^{i}$ is the strain energy in the materials after $k$ thermal shocks.

\section{Strain energy $U$}

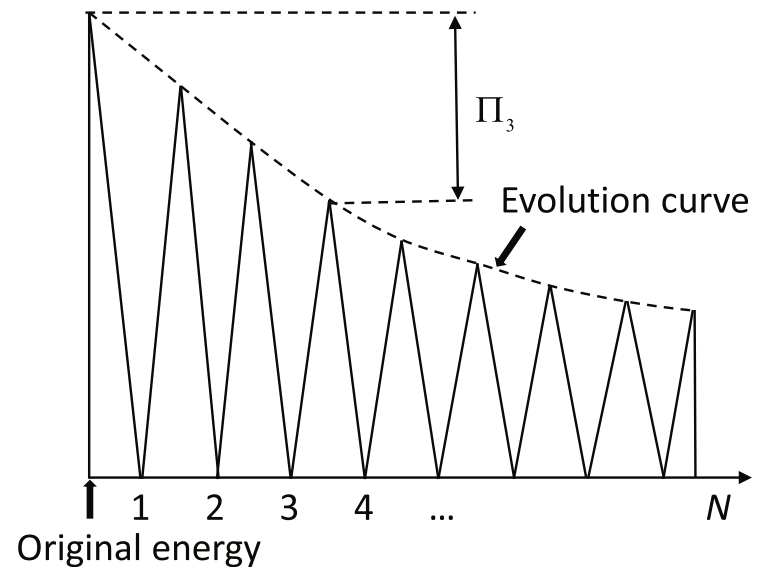

Fig. 7. Schematic of the energy dissipation evolution with the cumulative number of thermal shock cycles.

Then the total energy dissipation for a given shock temperature $T_{\text {shock }}$ can be derived as

$\Pi_{N}^{i}=\mathscr{U}_{0}-\mathscr{U}_{N}^{i}$.

As $\mathscr{U}_{N}^{i}$ decreases with thermal shock cycles $N, \Pi_{N}^{i}$ increases with the number of thermal shock cycles. Substituting Eq. (17) into Eq. (19), then

$\Pi_{N}^{i}=\mathscr{U}_{0}\left(2-D_{i}\right) D_{i}, \quad i=1,2,6$.

Eq. (20) illustrates the relationship between the total energy dissipation and the cyclic thermal shock-induced damage. As shown in Fig. 8, there is very good agreement between the model and the available experimental data, and the three directions of damage are on the same line, which indicates that the model essentially allows the prediction of damage development in the orthotropic CMCs.

\section{Numerical implementation}

A user-material subroutine (UMAT) was used to implement the proposed thermomechanical damage model of the ox/ox-CMCs under cyclic thermal shocks in the finite element industrial software ABAQUS/Standard. The geometrical dimensions of the finite element model are the same as those in the experiments. The simulation results are compared with the experimental data to verify the accuracy of the

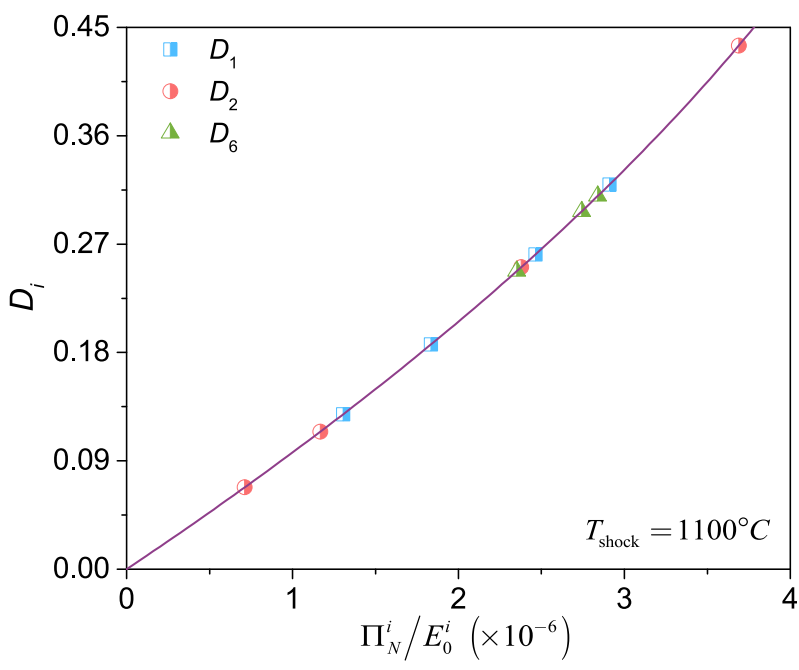

Fig. 8. Cyclic damage evolution with the dissipation energy $\Pi_{N}$. 


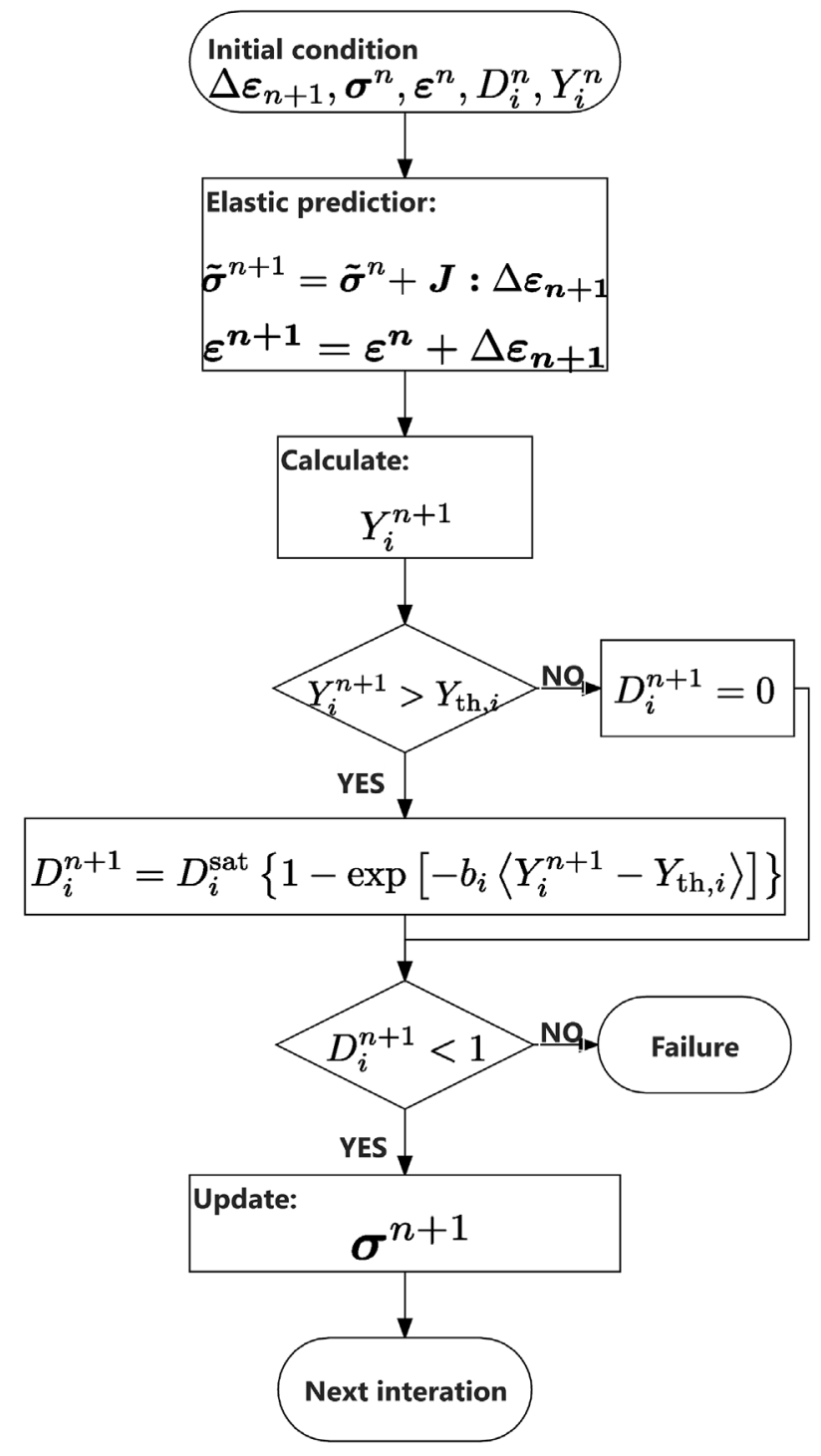

Fig. 9. Computational flow chart of the damage model for a single thermal shock.

proposed thermomechanical damage model.

In the cyclic thermal shock-induced thermomechanical damage model implementation processing, the incremental approach is driven by displacement. As numerical integrations over discrete time increments are requisite, the loading history is divided into a series of time steps $\left[t_{n}, t_{n+1}\right], n \in\{0,1,2, \ldots\}$, each step referring to the $(n+1)$ th increment. The backward Euler implicit integration algorithm based on the damage model is as follows:

$\left\{\begin{array}{l}\sigma_{n+1}=\boldsymbol{S}_{\mathrm{d}, n+1}: \varepsilon_{n+1} \\ \tilde{\sigma}_{n+1}=\boldsymbol{S}_{0}: \varepsilon_{n+1} \\ \varepsilon_{n+1}=\varepsilon_{n}+\Delta \varepsilon_{n+1},\end{array}\right.$

where $\varepsilon_{n}, \Delta \varepsilon_{n+1}$, and $\varepsilon_{n+1}$ are known quantities; $\varepsilon_{n+1}, \boldsymbol{S}_{\mathrm{d}, n+1}$, and $\sigma_{n+1}$ are unknown solution-dependent state variables to be solved. Fig. 9 is a computational flow chart of the thermomechanical damage model for the ox/ox-CMCs under a single thermal shock with different thermal shock temperatures $T_{\text {shock}}$. The computational flow for the ox/ox-CMCs under cyclic thermal shocks is similar to that presented previously.

Figs. 10 and 11 illustrate the results of computations compared with the experimental data of the ox/ox-CMCs under a single thermal shock with different thermal shock temperatures $T_{\text {shock }}$ and cyclic thermal shocks, respectively. The good agreement demonstrates the validity of

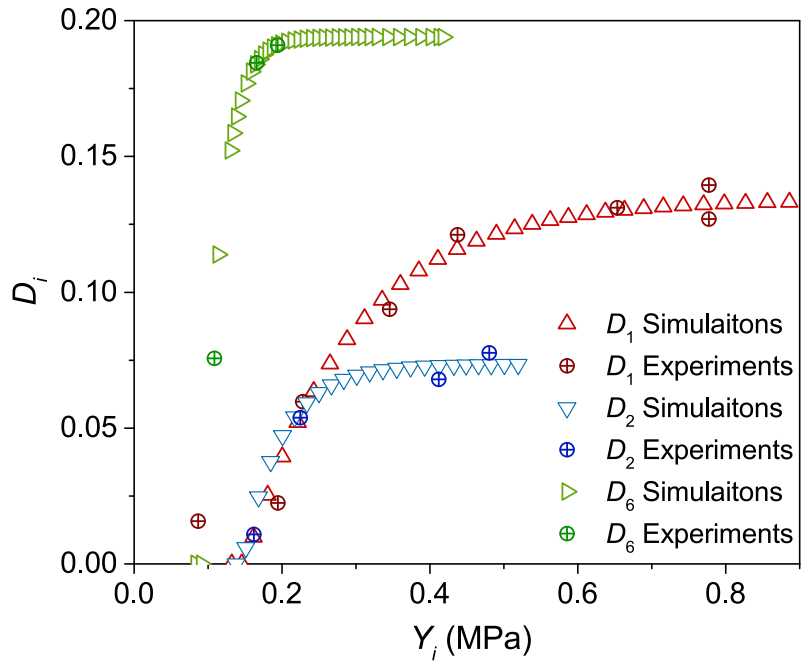

Fig. 10. Comparison of predicted and experimental damage of the ox/ox-CMCs with a single thermal shock.

the thermomechanical damage parameters obtained and substantiates those assumptions. More essentially, for the single thermal shock, Fig. 10 demonstrates that the thermal shock-induced thermomechanical damage increases rapidly with the strain energy density release rate $Y_{i}$, and when the thermal shock temperature exceeds $1000^{\circ} \mathrm{C}$, the thermal shock-induced thermomechanical damage goes into a saturation stage which is similar to the experimental results. This phenomenon in experiments may due to that the fiber reinforcements change the matrix crack propagation behavior and it also shows the fibers/matrices interfaces play an important role in the physical and mechanical properties of woven CMCs. To simulate the damage evolution process more accurately, it is necessary to consider the microstructure of composite materials during modeling in further work. In addition, the in-plane shear damage $D_{6}$ is much higher than that in other directions, which demonstrates that the thermomechanical damage in the matrix is serious, as the shear property of lamina is affected mainly by matrix. On the other hand, the simulated $D_{1}$ and $D_{2}$ show different saturation values, which may be caused by the influence of the specimen size and edge effect mentioned above. Besides, for the cyclic thermal shocks, Fig. 11 shows that the damage evolution are coincident with the experimental results for all orientations. Furthermore, when comparing the simulation results in three orientations, interesting is that the computed results and the experimental results are on the same line, indicating that the damage in the three orientations can be represented by the same evolution law, and the computed results have proved this phenomenon.

\section{Conclusions}

In the present work, a CDM model for the prediction of thermomechanical damage in ox/ox-CMCs under cyclic thermal shocks has been presented and analyzed, which is an extended version of the model proposed in previous work [25]. The originality of this model lies in the possibility to predict residual mechanical performance for anisotropic composites under cyclic thermal shocks. The model can be also applied to the damage tolerance analysis of CMC structures. Considering the detailed experimental and computational results, the following conclusions can be drawn.

- The damage mechanisms of the material under thermal shocks are delamination and micro-cracks, and the matrix crack density increases with the cumulative number of thermal shock cycles, which leads to the continuous degeneration of the material property. After only a limited number of thermal shock cycles, the rate of increase 


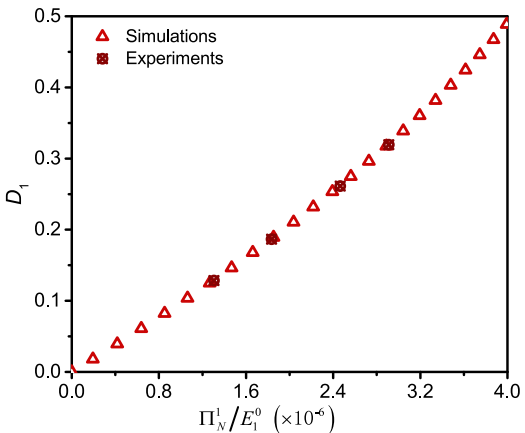

(a) Damage evolution of direction 1

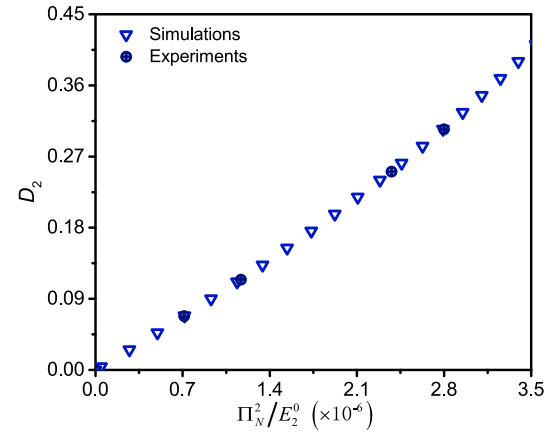

(b) Damage evolution of direction 2

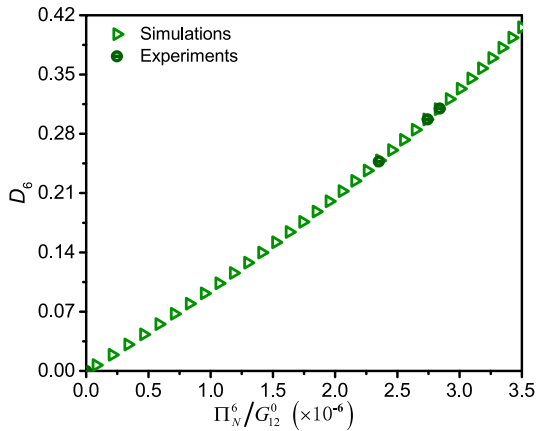

(c) Damage evolution of shear direction

Fig. 11. Comparison of predicted and experimental damage of the ox/ox-CMCs under cyclic thermal shocks.

in micro-crack density slows down and reaches a saturation level, because the matrix crack propagation is limited by the reinforced fibers.

- The residual stiffness of the investigated ox/ox-CMCs decreases gradually with increasing temperature/cumulative number of thermal shock cycles, and the effect of thermal shock on the residual mechanical properties in $0^{\circ} / 90^{\circ}$ and $\pm 45^{\circ}$ fiber orientations demonstrates the anisotropic behavior, indicating that the orthogonal material property in the ox/ox-CMCs under cyclic thermal shocks affects the degeneration of the modulus.

- An anisotropic thermomechanical damage evolution model of the ox/ox-CMCs has been proposed based on CDM, the identification of the damage parameters has been obtained by uniaxial off-axis and on-axis tensile tests. The internal mechanism of thermomechanical damage in the ox/ox-CMCs under cyclic thermal shocks can be interpreted reasonably and physically through strain energy, under the hypothesis that the damage occurs in the elastic stage.

Although the proposed thermomechanical damage model has been proved to perform well for standard specimens of ox/ox-CMCs, it is necessary for additional validations and developments. Work in this area is ongoing and will be discussed in future work.

\section{Acknowledgement}

The present work is supported by the Strategic Priority Research Program of Chinese Academy of Sciences (Grant No. XDA17030100), the National Natural Science Foundation of China (Grant No. 11572169 and 51775294).

\section{Appendix A. Supplementary data}

Supplementary data to this article can be found online at https:// doi.org/10.1016/j.ceramint.2019.11.060.

\section{References}

[1] Y. Gowayed, E. Abouzeida, I. Smyth, G. Ojard, J. Ahmad, U. Santhosh, G. Jefferson, The role of oxidation in time-dependent response of ceramic-atrix composites, Compos. B Eng. 76 (2015) 20-30.

[2] Dianyun Zhang, Pascal Meyer, Anthony M. Waas, An experimentally validated computational model for progressive damage analysis of notched oxide/oxide woven ceramic matrix composites, Compos. Struct. 161 (2017) 264-274.

[3] Z. Yang, H. Liu, Effects of thermal aging on the cyclic thermal shock behavior of oxide/oxide ceramic matrix composites, Mater. Sci. Eng. A 769 (2020), https://doi. org/10.1016/j.msea.2019.138494 138494.

[4] S. Askarinejad, N. Rahbar, V. Sabelkin, S. Mall, Mechanical behavior of a notched oxide/oxide ceramic matrix composite in combustion environment: Experiments and simulations, Compos. Struct. 127 (2015) 77-86.

[5] Seyed Mohammad Mahdi Zamani, Kamran Behdinan, Multiscale modeling of the mechanical properties of Nextel 720 composite fibers, Compos. Struct. 204 (2018) 578-586.

[6] Zhengmao Yang, Junjie Yang, Investigation of long-term thermal aged damage in oxide/oxide ceramic matrix composites, J. Eur. Ceram. Soc. (2019), https://doi. org/10.1016/j.jeurceramsoc.2019.10.052

[7] R.N. Singh, H.Y. Wang, Thermal-shock behavior of fiber-reinforced ceramic-matrix composites, Compos. Eng. 5 (10-11) (1995) 1287-1297.

[8] H.Y. Wang, R.N. Singh, R.A. Lowden, Thermal shock behavior of two-dimensional woven fiber-reinforced ceramic composites, J. Am. Ceram. Soc. 79 (7) (1996) 1783-1792.

[9] Yutaka Kagawa, Thermal shock damage in a two-dimensional SiC/SiC composite reinforced with woven sic fibers, Compos. Sci. Technol. 57 (5) (1997) 607-611.

[10] C. Kastritseas, P.A. Smith, J.A. Yeomans, Thermal shock fracture in unidirectional fibre-reinforced ceramic-matrix composites, Compos. Sci. Technol. 65 (11-12) (2005) 1880-1890.

[11] C. Kastritseas, P.A. Smith, J.A. Yeomans, Damage characterisation of thermally shocked cross-ply ceramic composite laminates, J. Mater. Sci. 41 (3) (2006) 951-962.

[12] Sirajus Salekeen, Justice Nana Amoako, Mahfuz Hassan, Shaik Jeelani, Mechanical property degradation of a nicalon fiber reinforced SiNC ceramic matrix composite under thermal shock loading, Compos. Struct. 78 (4) (2007) 477-485.

[13] Hui Mei, Laifei Cheng, Stress-dependence and time-dependence of the post-fatigue tensile behavior of carbon fiber reinforced SiC matrix composites, Compos. Sci. Technol. 71 (11) (2011) 1404-1409.

[14] L. Alma, Leanos and Pavana Prabhakar. Experimental investigation of thermal shock effects on carbon-carbon composites, Compos. Struct. 132 (2015) 372-383.

[15] A. Udayakumar, M. Stalin, M.B. Abhayalakshmi, Ramya Hariharan, M. Balasubramanian, Effect of thermal cycling of $\mathrm{SiC}_{\mathrm{f}} / \mathrm{SiC}$ composites on their mechanical properties, J. Nucl. Mater. 442 (1) (2013) S384-S389 Supplement 1.

[16] Chengyu Zhang, Mengmeng Zhao, Yongsheng Liu, Bo Wang, Xuanwei Wang, Shengru Qiao, Tensile strength degradation of a 2.5D-C/SiC composite under thermal cycles in air, J. Eur. Ceram. Soc. 36 (12) (2016) 3011-3019.

[17] K.I. Triantou, K. Mergia, B. Perez, S. Florez, A. Stefan, C. Ban, G. Pelin, G. Ionescu, C. Zuber, W.P.P. Fischer, J. Barcena, Thermal shock performance of carbon-bonded carbon fiber composite and ceramic matrix composite joints for thermal protection re-entry applications, Compos. B Eng. 111 (2017) 270-278.

[18] V. Vinogradov, Z. Hashin, Probabilistic energy based model for prediction of transverse cracking in cross-ply laminates, Int. J. Solids Struct. 42 (2) (2005) 365-392.

[19] U. Santhosh, J. Ahmad, G. Ojard, R. Miller, Y. Gowayed, Deformation and damage modeling of ceramic matrix composites under multiaxial stresses, Compos. B Eng. 90 (2016) 97-106.

[20] Qiang Yang, Xinxing Han, Chenghai Xu, Weihua Xie, Songhe Meng, Development and validation of an anisotropic damage constitutive model for c/sic composite, Ceram. Int. 44 (18) (2018) 22880-22889.

[21] J.B. Min, D. Xue, Y. Shi, Micromechanics modeling for fatigue damage analysis designed for fabric reinforced ceramic matrix composites, Compos. Struct. 111 (2014) 213-223.

[22] C. Chateau, L. Gélébart, M. Bornert, J. Crépin, Micromechanical modeling of the elastic behavior of unidirectional CVI SiC/SiC composites, Int. J. Solids Struct. 58 (2015) 322-334.

[23] J.L. Chaboche, J.F. Maire, New progress in micromechanics-based CDM models and their application to CMCs, Compos. Sci. Technol. 61 (15) (2001) 2239-2246.

[24] J.L. Chaboche, J.F. Maire, A new micromechanics based CDM model and its application to CMC's, Aero. Sci. Technol. 6 (2) (2002) 131-145.

[25] Z. Yang, H. Yuan, H. Liu, Evolution and characterization of cyclic thermal shockinduced thermomechanical damage in oxide/oxide ceramics matrix composites, Int J. Fatigue 120 (2019) 150-161.

[26] Z. Yang, H. Yuan, B. Markert, Representation of micro-structural evolution and thermo-mechanical damage in thermal shocked oxide/oxide ceramic matrix composites, Int. J. Fatigue 126 (2019) 122-129.

[27] Z. Yang, H. Liu, H. Yuan, Micro-porosity as damage indicator for characterizing cyclic thermal shock-induced anisotropic damage in oxide/oxide ceramic matrix composites, Eng. Fract. Mech. 220 (2019), https://doi.org/10.1016/j.engfracmech. 2019.106669 106669 .

[28] ASTM International, D3518/D3518M-18 Standard Test Method for In-Plane Shear Response of Polymer Matrix Composite Materials by Tensile Test of a $\pm 45^{\circ}$ Laminate, American Society for Testing and Materials (2018) Retrieved from https://doi.org/10.1520/D3518 D3518M-18. 
[29] Y. Gowayed, J. Pierce, D. Buchanan, L. Zawada, R. John, K. Davidson, Effect of microstructural features and properties of constituents on the thermo-elastic properties of ceramic matrix composites, Compos. B Eng. 135 (2018) 155-165.

[30] V. Kostopoulos, D.E. Vlachos, A. Paipetis, G. Sotiriadis, Anisotropic damage of alumina/alumina cfccs under long term high temperature exposure: Investigations by ultrasonic stiffness measurements and quasi-static tests, Compos. Sci. Technol. 66 (2006) 3221-3229.

[31] M.N. Rossol, V.P. Rajan, F.W. Zok, Effects of weave architecture on mechanical response of 2D ceramic composites, Compos. Appl. Sci. Manuf. 74 (Supplement C) (2015) 141-152.

[32] V.A. Lubarda, D. Krajcinovic, Damage tensors and the crack density distribution,
Int. J. Solids Struct. 30 (20) (1993) 2859-2877.

[33] Varun P. Rajan, John H. Shaw, Michael N. Rossol, Frank W. Zok, An elastic-plastic constitutive model for ceramic composite laminates, Compos. Appl. Sci. Manuf. 66 (2014) 44-57.

[34] G. Camus, Modelling of the mechanical behavior and damage processes of fibrous ceramic matrix composites: application to a 2-D SiC/SiC, Int. J. Solids Struct. 37 (6) (2000) 919-942.

[35] Z. Yang, L. Hui, J. Yang, Damage constitutive model for thermal shocked-ceramic matrix composite, Chin. J. Theor. Appl. Mech. 51 (6) (2019) 1-13.

[36] J. Lemaitre, R. Desmorat, Engineering Damage Mechanics: Ductile, Creep, Fatigue and Brittle Failures, Springer-Verlag Berlin Heidelberg, 2005. 